\title{
Kleihauer-Betke Staining Procedure
}

National Cancer Institute

\section{Source}

National Cancer Institute. Kleihauer-Betke Staining Procedure. NCI Thesaurus. Code C161409.

A biospecimen collection and staining procedure in which maternal blood smear is prepared and stained using Shepard's staining method, followed by microscopic examination to identify fetal-maternal hemorrhage (fetal hemoglobin transference to the mother's bloodstream). 